\title{
A HIGH-CONDUCTING STATE IN LIQUID METAL-AMMONIA SOLUTIONS
}

\author{
P. ARENDT \\ Institut für Physikalische Chemie und Elektrochemie, Universität Karlsnuhe, Karlsruhe, Germany
}

\begin{abstract}
Metal-ammonia solutions (1.5 to 5 mole per cent alkali metal) with added traces of water and oxygen can be transferred into a high-conducting state by a dc current forced through the solution with a critical voltage and critical power dissipation, respectively. This high-conducting state is stabilized by the viscous gel formed by the reaction of the metal with the added impurities.
\end{abstract}

\section{Introduction}

Solutions of $\mathrm{Li}, \mathrm{Na}$, and $\mathrm{K}$ in liquid ammonia with initial metal concentrations between 1.5 and 5 MPM (mole per cent metal) and temperatures between -40 and $-10{ }^{\circ} \mathrm{C}$ can be transferred into a high-conducting state /1-3/ if during their preparation they are exposed to the open air, i.e. allowing water vapour and oxygen to enter the solutions as impurities. One preparation procedure with which we can get the high-conducting state is the following: about $15 \mathrm{ml}$ ammonia are distilled into an open glass vessel $(3 \mathrm{~cm}$ diameter, $10 \mathrm{~cm}$ high). Alkali metal freshly cut under the free atmosphere is added. The cell is closed with a rubber stopper provided with four electrodes (see inset Fig. 1) to measure dc voltamograms of the solutions by the four pole technique. The two outer compact copper electrodes $(3 \mathrm{~mm}$ diameter, $1.4 \mathrm{~cm}$ distance) are connected with a dc-power supply (up to $100 \mathrm{~A}$ and $30 \mathrm{~V}$ ), the two inner electrodes $(0.4 \mathrm{~mm}$ diameter, $1 \mathrm{~cm}$ distance) serve to measure the voltage drop, when a current passes through the solution.

In the beginning the voltamograms are linear (as always found with highly purified metalammonia solutions), indicating normal ohmic conductance. The resistance of the solutions however increases due to the slow reaction of the dissolved metal with the added impurities (in contrast to common assumption, the reaction between sodium and water in ammonia takes up to ten hours to complete $/ 4 /$ ). Finally a critical state is reached in the solution: the voltamograms become nonlinear, they flatten, i.e. the differential conductance $\mathrm{dI} / \mathrm{dV}$ decreases with increasing voltage drop, and at a critical voltage drop $V_{c}$ and corresponding critical current $I_{c}$ the differential conductance approaches zero. This is the point of instability: the vol- 


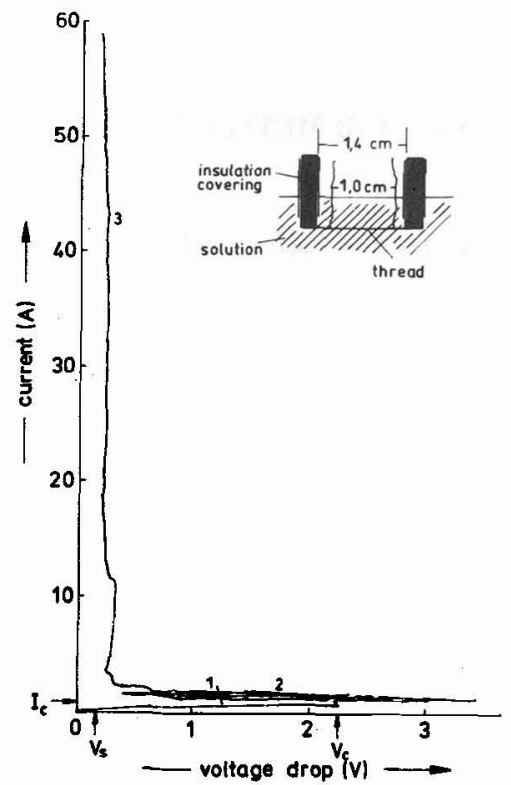

Fig. 1. - The normal-conducting regime 1, the instability regime 2 , and the high-conducting regime 3 for a $\mathrm{Na}-\mathrm{NH}_{3}$ solution with an initial concentration of $2.5 \mathrm{MPM}$ at $-40^{\circ} \mathrm{C}$, recorded with increasing current. Inset: geometrical arrangement of the four electrodes (to which a wool thread of 0.25 $\mathrm{mm}$ diameter is attached; compare chapter 2).

tage drop, starting from the critical voltage drop $v_{c}$, begins to jump back and forth at nearly constant current. Now, by slowly reducing the voltage applied externally, the solution jumps suddenly to the high-conducting state: the current first increases up to $10 \mathrm{~A}$ at constant voltage drop $V_{s}\left(\ll V_{c}\right.$ ), with the external voltage held constant (the current reached depends on the external circuit resistance). By increasing then the external voltage, currents of about $100 \mathrm{~A}$ can be reached at constant voltage drop $\mathrm{V}_{s}$, indicating almost zero differential resistance. With larger currents the high-conducting state will be destroyed, current and voltage drop jump back to the original values of the normal-conducting regime of the solutions. But from this state the solution can be again transferred to the high-conducting state. Currents of about 40 A can be sustained for hours.

The critical electrical values from which the system jumps into the high-conducting state depend (besides on the geometry) on the initial metal concentration of the solution. Using the geometry given above, $\mathrm{V}_{\mathrm{c}}$ decreases with increasing concentration ( 1.5 to 5 MPM) from about 2.5 to $0.5 \mathrm{~V}$ and $\mathrm{I}_{c}$ increases from about 0.6 to $3.0 \mathrm{~A}$; however, the product of the two, the dissipated electric power $\mathrm{I}_{c} \mathrm{~V}_{c}$, is about $1.5 \mathrm{~W}$.

In the high-conducting state the currents (up to $100 \mathrm{~A}$ ) can be sustained at a voltage drop $\mathrm{V}_{\mathrm{s}}$ of only 0.01 to $0.15 \mathrm{~V}$. There seems to be no correlation in the value of $\mathrm{V}_{\mathrm{s}}$ with the initial metal concentration. 
2. Additional remarks concerning the preparation of the solutions and the results obtained

The reaction of the impurities with the metal leads to a viscous gel-like product in which current-carrying capillaries enclosing non-decomposed solution are embedded. The radius of the capillaries of about $4 \times 10^{-4} \mathrm{~cm}$ has been determined by a technique which makes visible the patterns which the current engraves on the electrodes (for further details, see reference $/ 5 /$ ). The network of the gel may stabilize the capillaries against both disruption by thermal agitation and destabilization by diffusive flow of matter in and out of the capillaries. The capillaries, however, can further be stabilized by a porous insulating material (foam plastics, fritted glass, wool thread) placed between the current electrodes. Then the solutions can be transferred to the high-conducting state in $95 \%$ of the experiments. Without porous material, the reproducibility is much lower (about 20\%), the jump to the high-conducting state depends more critically on the amount of impurities taken up during the preparation of the solutions. The voltamogram in figure 1 was obtained with wool thread $(0.25 \mathrm{~mm}$ diameter) as porous insulator stretched between the electrodes.

The time required to reach the unstable state depends on the rate with which the gel-like state is "formed in the solution: if the current electrodes with a wool thread between them are kept in the cold solution (as in Fig. 1), some minutes may elapse (while recording repeatedly the voltamograms) until the differential conductance $\mathrm{dI} / \mathrm{dV}$ approaches zero along the normal-conducting regime 1 in figure 1.

The gel forms faster and hence the high-conducting regime is reached faster with the following experimental method: a 1.6 MPM Na-solution is prepared as described before. After a contact time of a few seconds the electrode arrangement with the wool thread is pulled out of the solution $\left(-25^{\circ} \mathrm{C}\right)$ and kept in the ammonia vapour phase above it (1.5 bar). The voltamogram taken immediately after this is shown in figure 2. Due to the current heat dissipated in the minute amount of solution on the thread the formation of the gel is accelerated,

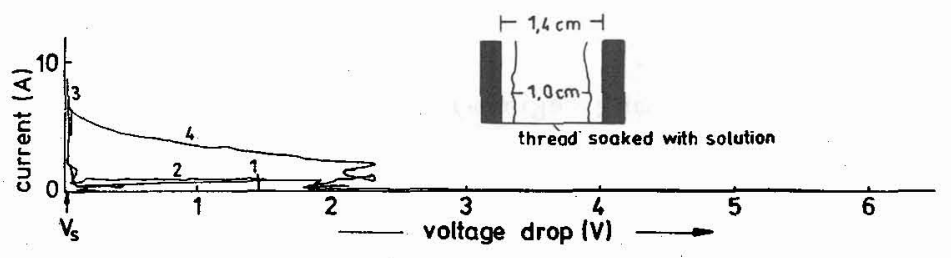

Fig. 2. - Voltamogram of a 1.6 MPM sodium-ammonia solution sucked up by a thread of $0.25 \mathrm{~mm}$ diameter. The current successively runs along the normal-conducting regime 1 , the instability regime 2 , the high-conducting regime 3 , and finally jumps along 4 to a value which corresponds to the high resistance of the boiled down solution on the thread. 
and the system passes successively within a few seconds from the normal ohmic behaviour via the instability state $(\mathrm{dI} / \mathrm{dV} \simeq 0$ ) to the high-conducting regime (reaching there currents up to $10 \mathrm{~A}$ at a voltage drop of $\mathrm{V}_{\mathrm{s}} \simeq 0.01 \mathrm{~V}$, corresponding to a resistance of $1 \times 10^{-3} \Omega$ ). However after another few seconds, the high-conducting state will be destroyed, current and voltage drop jumping to values which correspond to the high resistance of the boiled down solution taken up by the thread.

The initial metal concentrations between 1.5 and 5 MPM have been chosen for the following reasons: solutions with concentrations below 1.5 MPM do not form the proper gel-like state. They decompose to a dirty greasy substance which usually does not become high-conducting, even if some porous material is placed between the electrodes. The solutions with concentrations above S MPM usually do not become high-conducting because the critical electric power $\mathrm{I}_{c} \mathrm{~V}_{c}$ (about $1.5 \mathrm{~W}$ necessary at an interelectrode distance of $1 \mathrm{~cm}$ ) is dissipated already at voltages which are about equal or even below the voltage drop $v_{s}$ at which the high-conducting state is sustained.

The experiments were carried out above $-40{ }^{\circ} \mathrm{C}$ to avoid the miscibility gap of metalammonia solutions and below $-10^{\circ} \mathrm{C}$ to avoid vapour pressures above 3 bars within the glass vessel.

The formation of the viscous gel in the solutions is induced by the presence of impurities: in some experiments water has been added in a known quantity $(0.1$ vol\%), however, the amounts of oxygen, carbon dioxide, and additional water condensed on the inner walls of the vessel, that have entered the solution from the free atmosphere, are unknown. Likewise we do not know the amount of oxides on the alkali metal freshly cut under the free atmosphere before being added. Nevertheless, with the preparation procedure described before the high-conducting state can be reproducibly obtained, the high-conducting state does not depend critically on the concentrations of the impurities within the limits of uncertainty due to the preparation procedure used. However, the variation of the sustaining voltage drop $V_{s}$ (within the limits 0.01 and $0.15 \mathrm{~V}$ ) may be due to the variation in the concentrations of the impurities added.

\section{Dịscussion}

To test whether the high-conducting regime is due to an enrichment of dissolved metal in the space between the electrodes or even due to the formation of a metallic filament, we compared the resistance $\left(1 \times 10^{-3} \Omega\right)$ in the high-conducting state of the metal solution impregnated on the wool thread (as described before) with the calculated resistance of a fictive filament made up from the metal which was taken up by the thread: the amount of sodium on the thread taken up from the 1.6 MPM solution is about $3 \times 10^{-5} \mathrm{~g}$. It was determined by 
titration, after the thread had been transferred from the solution to water in which the sodium is decomposed to sodium hydroxide (the ammonia was boiled off from the aqueous solution). This sodium could form a filament between the electrodes of about $3 \times 10^{-5} \mathrm{~cm}^{2}$ in cross-section and $1 \mathrm{~cm}$ in length (= interelectrode distance) and hence would have a resistance of about $0.1 \Omega$. Because in our experiments the conductance of the impregnated wool thread is by a factor of $10^{2}$ larger than the conductance of the fictive filament, we conclude that the observed high conductivity probably has a non-trivial reason, i.e. that we really deal with a new high-conducting state.

Acknowledgements

The author would like to thank Prof. Dr. U. Schindewolf for many fruitful discussions. Financial support by the Deutsche Forschungsgemeinschaft is gratefully acknowledged.

References

1/ A rendt, P., Z. Phys. Chem. (Leipzig) 263 (1982) 689; Electrochim. Acta 30 (1985) 709; J. Phys. Chem. Solids 49 (1988) S11; Solid State Commun. 67 (1988) 1167.

/2/ Dmitrenko, I.M., Moshenskii, A.A., and Revvo, B.L., Sov. J. Low Temp. Phys. 10 (1984) 449; Vasilchenko, S.A., Dmitrenko, I.M., and Moshenskii, A.A., Sov. J. Low Temp. Phys. 11 (1985) 119.

/3/ Burkart, R., Diplomarbeit, Universität Karlsruhe (1990).

/4/Telser, Th. and Schindewolf, U., Ber. Bunsenges. Phys. Chem. 88 (1984) 488.

/5/ Arendt, P., Solid State Commun. 70 (1989) 1001; 74 (1990) 559. 